\title{
Bowel preparation in diabetic patients undergoing colonoscopy
}

\author{
Osama Qasim Agha ${ }^{a, b}$, Muhammad Alsayid', Michael D. Brownc
}

Creighton University School of Medicine, Phoenix, Arizona; St Joseph's Hospital and Medical Center, Phoenix, Arizona; Rush University Medical Center, Chicago, Illinois, USA

\begin{abstract}
Diabetes mellitus is a risk factor for poor bowel preparation in patients who undergo colonoscopy, because of their decreased intestinal transit and slow gastric emptying. This might lead to neoplastic or preneoplastic lesions being missed, longer procedural time, a higher risk of procedurerelated adverse events, significant cost burden, patient dissatisfaction, and the need for a repeat colonoscopy. Multiple strategies have been suggested to improve bowel preparation in these patients. Proposed pharmacologic strategies include adding magnesium citrate, bisacodyl, lubiprostone or pyridostigmine. Non-pharmacologic strategies include preferential procedure scheduling or using a diabetes-specific preparation protocol. In this article, we present a comprehensive review of the literature and provide specific recommendations to general practitioners and gastroenterologists for improving bowel preparation in patients with diabetes.
\end{abstract}

Keywords Colonoscopy, colorectal cancer, diabetes mellitus, bowel preparation

Ann Gastroenterol 2021; 34 (2): 1-6

\section{Introduction}

Colonoscopy is a routine gastrointestinal diagnostic and therapeutic procedure that provides a direct visualization of the colonic lumen. It can be used for the diagnosis and management of different colonic disorders as well as screening for colorectal cancer (CRC) [1]. The American Society of Gastrointestinal Endoscopy (ASGE) guidelines consider colonoscopy as a firsttier test for CRC screening, and about 17 million colonoscopies are performed annually in the United States (US) [2]. A complete colonoscopy requires cecal intubation and adequate preparation in order to visualize the entire colonic mucosa $[3,4]$. Patients with inadequate bowel preparation should undergo a repeat

${ }^{a}$ Division of Internal Medicine, Creighton University School of Medicine, Phoenix, Arizona (Osama Qasim Agha); ${ }^{\mathrm{b}}$ Division of Internal Medicine, St Joseph's Hospital and Medical Center, Phoenix, Arizona (Osama Qasim Agha); 'Division of Digestive Diseases, Rush University Medical Center, Chicago, Illinois (Muhammad Alsayid, Michael D. Brown), USA

Conflict of Interest: None

Funding: MA was supported by the National Center for Advancing Translational Sciences of the National Institutes of Health under Award Number TL1TR002388. The content is solely the responsibility of the authors and does not necessarily represent the official views of the National Institutes of Health

Correspondence to: Osama Qasim Agha, MD, 350 W Thomas Rd, Phoenix, AZ 85013, USA, e-mail: osmagha@gmail.com

Received 23 September 2020; accepted 12 January 2021; published online 26 February 2021

DOI: https://doi.org/10.20524/aog.2021.0599 colonoscopy within 1 year [4]. Diabetic patients are at greater risk of having inadequate bowel perpetration, which can lead to missed neoplastic or preneoplastic lesions, longer procedural time, a greater risk of procedure-related adverse events, significant cost burden, and patient dissatisfaction $[1,3,5]$. The risk of inadequate bowel preparation in diabetic patients has been attributed to several factors, and physicians' awareness of possible interventions can mitigate the risk of inadequate bowel preparation in these patients.

\section{Diabetes mellitus (DM) and bowel preparation}

DM has been found in multiple studies to be an independent risk factor for inadequate bowel preparation for colonoscopy. A systematic review and meta-analysis of 67 studies by Gandhi et al showed that DM had an odds ratio (OR) of 1.79 for inadequate bowel preparation (95\% confidence interval [CI] 1.54-2.09; $\mathrm{P}=0.001 ; I^{2}=54 \%$ ) [6]. Similarly, another systematic review and meta-analysis by Mahmood et al reported that DM was a risk factor for inadequate bowel preparation (OR 0.58, 95\%CI 0.43$\left.0.79 ; \mathrm{P}<0.001 ; I^{2}=85 \%\right)$ [7]. Sensitivity analysis showed that DM was associated with inadequate bowel preparation, whether split-dose (OR 0.67, 95\%CI 0.48-0.92; $\mathrm{P}=0.01$ ) or conventional bowel preparation (OR 0.43, 95\%CI $0.24-0.80$; $\mathrm{P}<0.001$ ) was used. In addition, sensitivity analysis showed that DM was significantly associated with poor bowel preparation in studies performed in western countries (OR 0.64, 95\%CI 0.49-0.82; $\mathrm{P}<0.001$ ), while studies performed in Asian countries did not show an association (OR 0.46, 95\%CI 0.15-1.45; $\mathrm{P}=0.19$ ). This difference between the 2 populations might be explained by the 
differences in dietary habits, as Asian populations are thought to have more fibers and spicy food in their diet [7]. In addition, the differences in the prevalence of other risk factors for poor bowel preparation might also play a role [7].

The exact mechanism and pathophysiology behind inadequate bowel preparation in diabetic patients remain incompletely understood. However, it has been suggested that decreased intestinal transit and slow gastric emptying may play a major role [8]. These gastrointestinal dysfunctions may be due to changes in the microenvironment of the autonomic nervous system, the enteric nervous system and the intestinal cells of Cajal [9]. These changes include increased oxidative stress due to an excess of reactive oxygen species, a reduction in growth factors, changes in enteric microbiota, and alterations in signaling pathways and endothelial dysfunction [9].

Multiple studies have evaluated diabetic patients' characteristics to determine the risk factors for inadequate bowel preparation (Table 1). Some of these studies were small and the results were mostly inconsistent. Four of these studies showed that hemoglobin A1C had no significant relationship with the quality of bowel preparation [10-13]. Body mass index also had no significant relationship with the quality of bowel preparation, according to 3 studies [12-14]. A higher level of fasting plasma glucose was significantly associated with poorer bowel preparation in 2 small studies $[12,14]$.

The role of hypoglycemic medications in causing poor bowel preparation in diabetic patients has still not been widely investigated. Glucagon-like peptide 1 (GLP-1) agonists are commonly used hypoglycemic medications known to prolong gastrointestinal transit time [15]. However, a retrospective study by Sharma et al found no significant association between the use of GLP-1 agonists and poor bowel preparation [15].

Dietary habits might have an effect on colonic transit time and, in turn, on the quality of bowel preparation in diabetic patients. This might explain why DM was associated with poor bowel preparation in western countries compared to Asian countries, based on the meta-analysis mentioned above [7]. It might also explain the improvement in bowel preparation associated with adopting a special menu in the Alvarez study, which will be detailed later in this article [16].

\section{Pharmacologic interventions for improving bowel preparation in diabetics}

Several interventions have been studied or suggested to improve the quality of bowel preparation in diabetic patients (Tables 2,3). However, the absence of specific society guidelines and the lack of strong and consistent evidence have left patients and clinicians in a dilemma. The US Multi-Society Task Force on CRC recommended considering additional bowel purgatives in patients with risk factors for inadequate bowel preparation [5]. The ASGE also recommends that intensive education and bowel preparation regimens more aggressive than the standard ones should be considered when a risk factor for inadequate bowel preparation exists [3]. In the following paragraphs, we will review studies that have attempted to implement or suggest interventions that might improve the quality of bowel preparation in diabetic patients.

\section{Lubiprostone}

Lubiprostone is a chloride channel activator that increases fluid secretion in the intestines and, in turn, increases fecal transit [5]. Lubiprostone is approved for use in patients with chronic constipation and also for patients who suffer from irritable bowel syndrome with constipation [17]. While lubiprostone is not routinely given as a part of bowel preparation prior to colonoscopy, multiple studies have assessed its efficacy

Table 1 Summary of risk factors for inadequate bowel preparation in diabetic patients

\begin{tabular}{|c|c|c|c|c|c|}
\hline Factors & $\begin{array}{l}\text { Taylor et al. } 2001 \\
\qquad(\mathrm{~N}=45)[10]\end{array}$ & $\begin{array}{l}\text { Ozturk et al. } 2010 \\
\qquad(\mathrm{~N}=50)[14]\end{array}$ & $\begin{array}{l}\text { Izzy et al. } 2016 \\
(\mathrm{~N}=352)[11]\end{array}$ & $\begin{array}{l}\text { Kim et al. } 2017 \\
\quad(\mathrm{~N}=50)[12]\end{array}$ & $\begin{array}{l}\text { Lankalapalli et al. } 2017 \\
\qquad(\mathrm{~N}=380)[13]\end{array}$ \\
\hline Age & NS & $\mathrm{P}=0.0001$ & - & NS & NS \\
\hline Sex & - & NS & - & NS & $\mathrm{P}=0.02$ \\
\hline BMI & - & NS & & NS & NS \\
\hline A1C & NS & $\mathrm{P}=0.04$ & NS & NS & NS \\
\hline FPG & - & $\mathrm{P}=0.01$ & - & $\mathrm{P}=0.016$ & - \\
\hline MBGC & - & - & - & - & NS \\
\hline Duration of DM & - & $\mathrm{P}=0.009$ & - & NS & - \\
\hline Insulin use & NS & - & - & - & - \\
\hline Oral hypoglycemics & NS & - & - & - & - \\
\hline Late complications & - & $\mathrm{P}=0.001$ & - & NS & - \\
\hline Peripheral neuropathy & NS & - & - & NS & - \\
\hline Autonomic neuropathy & - & - & - & NS & - \\
\hline
\end{tabular}

DM, diabetes mellitus; BMI, body mass index; FPG, fasting plasma glucose; MBGC, mean blood glucose concentration; NS, not statistically significant; (-), not addressed/reported 
Table 2 Evidence-based summary of interventions suggested to improve bowel preparation in patients with diabetes

\begin{tabular}{ll}
\hline Interventions & Summary of evidence \\
\hline Lubiprostone & - Mixed results in non-diabetic patients \\
& - Insignificant favorable effect based on one \\
& study in diabetic patients \\
& - More studies are needed to evaluate its \\
& efficacy
\end{tabular}

PEG, polyethylene glycol; ASGE, American Society of Gastrointestinal Endoscopy; PEG-ELS, polyethylene glycol electrolyte lavage solution; DSP, diabetes-specific preparation

when added to polyethylene glycol (PEG) solution, with mixed results [18-20]. Grigg et al enrolled 60 diabetic patients in a single-blinded randomized trial that compared giving 2 doses of lubiprostone $(24 \mu \mathrm{g})$ in addition to a single dose of PEG ( $2 \mathrm{~h}$ prior to PEG and $2 \mathrm{~h}$ after PEG completion) versus giving PEG alone [17]. While the study showed more favorable results in the group that received lubiprostone, the results were not statically significant. The study was stopped early because of loss of funding, which resulted in a small sample size. Therefore, there is no strong evidence to support the routine use of lubiprostone to improve bowel preparation in diabetic patients.

\section{Magnesium citrate}

Magnesium citrate is an over-the-counter hyperosmotic laxative that acts by causing osmotic intraluminal fluid and electrolyte retention, leading to increased peristalsis of the small intestines and the colon [3]. Magnesium citrate is not approved by the Food and Drug Administration as a colonoscopy preparation agent and there is limited evidence about its efficacy as a standalone agent. Hayes et al performed a singleblinded, randomized controlled trial involving 198 diabetics, mostly male patients, undergoing outpatient colonoscopy to evaluate the efficacy of adding magnesium citrate to the standard bowel preparation [21]. There was significantly better bowel preparation in patients who received magnesium citrate plus PEG compared to patients who received PEG alone (70\% vs. $54 \% ; \mathrm{P}=0.02)$. The overall rate of good bowel preparation was lower than desired because of the use of single dose PEG in both groups, not supported by the current ASGE guidelines [3].

While Hayes et al reported no significant adverse effects in their study, the ASGE is currently recommending against the routine use of magnesium citrate in preparation for colonoscopy, given its potential side effects and the limited data related to its use [3]. The ASGE recommends against its use in patients with chronic kidney disease, a common complication of DM [3]. In addition, the ASGE recommends avoiding it in elderly patients, patients with congestive heart failure or decompensated cirrhosis, or patients taking medications that alter renal blood flow or electrolyte excretion $[3,22]$.

\section{Bisacodyl}

Bisacodyl is a prokinetic laxative derived from diphenylmethane. Its active metabolite works mainly on the colon, as it is poorly absorbed in the small intestines [23]. Bisacodyl has been used for the treatment of constipation since 1952, and it has also been used to reduce the volume of lavage solution used prior to colonoscopy $[3,23]$. In a singleblinded, randomized controlled trial by Madhoun et al, 212 adult diabetic patients undergoing outpatient colonoscopy were randomized to 3 intervention groups: split-dose 4 L PEG electrolyte lavage solution (PEG-ELS) preceded by $10 \mathrm{mg}$ of oral bisacodyl (SDBP-B), split-dose 4 L PEG-ELS (SDBP) and a single dose of 4 L PEG-ELS (CDP) [1]. The primary outcome was adequate bowel preparation quality, defined as a Boston Bowel Preparation Scale score $\geq 6$ with no segmental score $<2$, or subjective rating by the endoscopist as "good" or "excellent". The primary outcome was highest in the SDBP group (83.3\%) followed by the SDBP-B group (74.6\%) and the CDP group (66.7\%). However, the results were not statistically significant $(\mathrm{P}=0.1)$. This study, performed at a veteran's affairs hospital with a predominantly male population, shows that adding bisacodyl to the split-dose bowel preparation is unlikely to improve the quality of bowel preparation in diabetic patients. The results of this study are consistent with the general impression that adding prokinetic agents to bowel preparation does not improve bowel cleansing [3]. 


\section{Pyridostigmine}

Neostigmine and pyridostigmine are acetylcholinesterase inhibitors that can increase acetylcholine levels at the synapses, which can lead to increased colonic motility. Neostigmine has been used for the treatment of acute colonic pseudo-obstruction (Ogilvie syndrome). Pyridostigmine, in a small study, has been found to increase colonic transit and improve bowel function in diabetic patients with chronic constipation [24]. Pyridostigmine bromide was suggested for use in bowel preparation in diabetic patients with constipation [25]. However, there are no studies to support the efficacy or safety of pyridostigmine in bowel preparation for colonoscopy in either diabetic or non-diabetic patients. Moreover, the addition of prokinetic medications in general is not thought to be useful in improving bowel cleansing or patient tolerance [3].

\section{Glycemic control}

While DM is a known factor that can affect the quality of bowel preparation, it is unclear if attempting to achieve glycemic control prior to colonoscopy would provide any benefit. A retrospective study of 352 diabetic patients by Izzy et al showed that there was no significant relationship between the quality of preparation or adenoma detection and A1C levels [11]. Another retrospective study of 380 diabetic patients by Lankalapalli et al showed that glycemic control based on recent $\mathrm{A} 1 \mathrm{C}$ level and mean blood glucose concentration had no significant effect on bowel preparation quality [13].

\section{Procedure timing}

There have been multiple studies that investigated the effect of the colonoscopy timing on the adequacy of the bowel preparation and adenoma detection rate (ADR), with variable results [26]. One retrospective study reported that afternoon colonoscopies had a higher rate of inadequate bowel preparation and 2 other studies reported a better ADR with morning colonoscopies [26-28]. Endoscopist fatigue was suggested to be a factor influencing the lower ADR in later colonoscopies [26,29]. Nevertheless, other studies have found no significant association between colonoscopy timing and ADR [30-32].

Current ASGE guidelines recommend split-dose bowel preparation, with the second dose ideally beginning 4-6 h before the time of colonoscopy, with completion at least $2 \mathrm{~h}$ before the procedure time [3]. This is challenging and usually inconvenient to patients undergoing early morning colonoscopies, as it would require the patients to wake up very early to take the second dose.

Diabetic patients are usually advised to schedule their surgeries in the early morning to avoid prolonged fasting and subsequent hypoglycemia or hyperglycemia [33-35]. However, there is no strong evidence to support this early scheduling [36]. In addition, the American Society of Anesthesiologists allows the intake of clear liquid diet up to a minimum of $2 \mathrm{~h}$ prior to a scheduled procedure under sedation or general anesthesia [37]. Therefore, allowing patients to have a clear liquid diet up to $2 \mathrm{~h}$ before later colonoscopies might decrease the risk of hypoglycemia. A limiting factor for this practice is the possibility of the patient having undiagnosed gastroparesis, which might require a longer period of fasting.

Only one study investigated the effect of colonoscopy timing on bowel preparation quality in diabetic patients [38]. In this cohort study, the investigators preferentially scheduled patients with DM to have screening colonoscopies at 9:30 am or later. All patients (diabetics and non-diabetics) received split dose 4-L PEG preparation. The patients scheduled at 9:30 am or later received the second dose $5 \mathrm{~h}$ before their appointments, while patients scheduled before 9:30 am received all bowel preparation on the day before their colonoscopies. All patients were instructed to start a low-fiber diet 4 days before the procedure and then switch to a clear liquid diet on the day prior to the procedure. Investigators then compared data obtained from colonoscopies performed after starting this preferential scheduling with those carried out previously. Investigators concluded that the preferential scheduling allowed more adequate bowel preparation in diabetic patients while not disadvantaging other non-diabetic patients. Hypoglycemic or hyperglycemic events were not reported. The results of this study contradict other studies done in the general population (diabetic and non-diabetic patients) and showed either a worse outcome or no difference when colonoscopies were scheduled at later times. In addition, there was a huge difference in the time intervals compared in this study (before 9:30 am versus after 9:30 am) and this places a question mark on the results and the applicability of this study.

\section{DM-specific preparation protocol}

Alvarez et $\mathrm{al}$, in a randomized controlled superiority trial, were the first ones to develop a multifactorial diabetic-specific preparation (DSP) protocol to improve bowel preparation for colonoscopy in diabetic patients. The DSP protocol included a face-to-face educational visit at the hospital with a qualified nurse. It also included a specific dietary plan developed by an endocrinologist and a registered dietitian, consisting of a 4-day menu that specified low-fiber carbohydrate intake in each meal, while a liquid diet was limited only to $8 \mathrm{~h}$ before the procedure. Lastly, the DSP protocol also included instructions for adjustment of blood glucose-lowering agents.

In contrast, the control group received conventional bowel preparation protocol (CBP): no educational visit, low-fiber diet for 3 days and then clear liquid diet for $24 \mathrm{~h}$ before colonoscopy; lastly, no specific recommendations were given to adjust blood glucoselowering agents. Both groups, DSP and CBP, received split-dose 4-L PEG solution. Inadequate bowel preparation, defined as a Boston Bowel Preparation Scale score $<2$ in any segment, was 20\% in the $\mathrm{CBD}$ group compared with $7 \%$ in the DSP group $(\mathrm{P}=0.014$; risk ratio $3.1,95 \% \mathrm{CI} 1.2-8$ ). However, there was no significant difference in cecal intubation rate or in ADR between the 2 groups. The authors argued that the association between the quality of bowel preparation and ADR has not been firmly established. 
Table 3 Major studies addressing bowel preparation in diabetic patients

\begin{tabular}{|c|c|c|c|c|}
\hline Study (Year) [Ref] & Size & Intervention & Type & Limitations \\
\hline Alvarez et al (2016) [16] & 150 & DSP protocol & $\begin{array}{l}\text { Single-blinded, single-center, } \\
\text { superiority RCT }\end{array}$ & $\begin{array}{l}\text { - Single-center study } \\
\text { - Might not be applicable in countries with } \\
\text { limited resources }\end{array}$ \\
\hline Grigg et al (2010) [17] & 60 & Lubiprostone & $\begin{array}{l}\text { Single-blinded, single-center } \\
\text { RCT }\end{array}$ & $\begin{array}{l}\text { - Single-center study } \\
\text { - Small size } \\
\text { - Used single-dose PEG solution }\end{array}$ \\
\hline Hayes et al (2011) [21] & 198 & $\begin{array}{l}\text { Magnesium } \\
\text { citrate }\end{array}$ & $\begin{array}{l}\text { Single-blinded, single-center } \\
\text { RCT }\end{array}$ & $\begin{array}{l}\text { - Single-center study } \\
\text { - Used single-dose PEG solution } \\
\text { - Small sample of women }\end{array}$ \\
\hline Hilsden et al (2017) [38] & $\begin{array}{l}34,415 \\
(1,805 \text { diabetics })\end{array}$ & $\begin{array}{l}\text { Preferential } \\
\text { scheduling }\end{array}$ & Cohort study & $\begin{array}{l}\text { - A cohort study design } \\
\text { - Wide range of time interval in the DM } \\
\text { group } \\
\text { - DM-related events were not reported }\end{array}$ \\
\hline Izzy et al (2016) [11] & 352 & $\begin{array}{l}\text { Glycemic } \\
\text { control }\end{array}$ & Retrospective study & $\begin{array}{l}\text { - Retrospective design } \\
\text { - Did not evaluate other confounders } \\
\text { - Used single-dose PEG solution }\end{array}$ \\
\hline Madhoun et al (2018) [1] & 212 & Bisacodyl & $\begin{array}{l}\text { Endoscopist-blinded, } \\
\text { single-center RCT }\end{array}$ & $\begin{array}{l}\text { - Single-center study } \\
\text { - Small sample of women }\end{array}$ \\
\hline
\end{tabular}

DSP, diabetes-specific preparation; $R C T$, randomized controlled trial; PEG, polyethylene glycol; DM, diabetes mellitus

While it is unclear which arm of the multifactorial DSP protocol had the largest impact on improving bowel preparation, it is possible that the dietary recommendation of a low-fiber diet had some role in improving bowel preparation. A clear liquid diet had been commonly advised for the day before the colonoscopy and it is unclear whether this practice provided any significant benefit to bowel preparation or tolerability [3]. Growing evidence from multiple studies suggests that a more liberalized diet (low-residue diet) on the day before colonoscopy provides better tolerability and better or similar bowel preparation quality [5]. Current ASGE guidelines recommend a low-residue diet before colonoscopy, whereas the US Multi-Society Task Force on CRC recommends either low-residue or full-liquid diet until the evening on the day before colonoscopy $[3,5]$.

Applying the DSP protocol might be difficult, especially in areas or countries with limited resources. A successful DSP intervention would require using a food menu derived from the available food in the local markets. The menu should also contain food options that are affordable, have acceptable flavors, and are within the local religious food restrictions. This will require consulting with local dietitians, endocrinologists and local market experts. In addition, the menu might need to be translated into multiple languages if they are spoken in the community. Lastly, finding qualified nurses familiar with the local dietary habits is also an integral part of the process.

\section{Concluding remarks}

$\mathrm{DM}$ is associated with suboptimal bowel preparation for colonoscopy. Several studies attempted to optimize bowel preparation in these patients. However, these studies vary in the strength of their evidence, and most of them did not use split-dosing regimens, which are part of the current ASGE recommendation (Table 3). While the DSP protocol used in the study of Alvarez et al seems to offer the best intervention published to date, more studies are needed to confirm the results of this study and compare this intervention to other optimized preparations. Additionally, studies are needed to identify risk factors for inadequate bowel preparation in diabetic populations and possibly intervene to mitigate these factors.

Taking into consideration the limitations of the available evidence, we recommend the following interventions: (a) counseling diabetic patients about the possibility of having an inadequate bowel preparation; (b) extensive counseling from well-trained nurses (or other qualified healthcare professionals) about preparation intake, dietary recommendations and specific adjustments of antihyperglycemic agents should be offered to all diabetic patients. Instructions should be provided in an oral and a written fashion in the patients' preferred language; (c) split dosing is recommended for all diabetic patients. Avoidance of magnesium citrate and sodium phosphate is also recommended, given the prevalence of renal disease in this population; (d) adding prokinetics such as bisacodyl to a bowel preparation regimen is discouraged, given the lack of evidence of any benefit and the possible harm; (e) consider recommending low-residue diets to all diabetic patients on the days before colonoscopy; and (f) consider consulting with the patient's primary care provider or endocrinologist, especially if the patient is taking multiple antihyperglycemic agents.

\section{References}

1. Madhoun MF, Chaudrey KK, Chisholm SS, Ahmed A, Frost B, Tierney WM. Efficacy and tolerability of various bowel preparations 
in diabetic patients: a randomized controlled trial. Endosc Int Open 2018;6:E1157-E1163.

2. An astounding 19 million colonoscopies are performed annually in the United States - iData Research. 2018. Available from: https:// idataresearch.com/an-astounding-19-million-colonoscopies-areperformed-annually-in-the-united-states/ [Accessed 15 February 2021].

3. Saltzman JR, Cash BD, Pasha SF, et al; ASGE Standards of Practice Committee. Bowel preparation before colonoscopy. Gastrointest Endosc 2015;81:781-794.

4. Rex DK, Schoenfeld PS, Cohen J, et al. Quality indicators for colonoscopy. Gastrointest Endosc 2015;81:31-53.

5. Johnson DA, Barkun AN, Cohen LB, et al; US Multi-Society Task Force on Colorectal Cancer. Optimizing adequacy of bowel cleansing for colonoscopy: recommendations from the US multi-society task force on colorectal cancer. Gastroenterology 2014;147:903-924.

6. Gandhi K, Tofani C, Sokach C, Patel D, Kastenberg D, Daskalakis C. Patient characteristics associated with quality of colonoscopy preparation: a systematic review and meta-analysis. Clin Gastroenterol Hepatol 2018;16:357-369.

7. Mahmood S, Farooqui SM, Madhoun MF. Predictors of inadequate bowel preparation for colonoscopy: a systematic review and metaanalysis. Eur J Gastroenterol Hepatol 2018;30:819-826.

8. Piper MS, Saad RJ. Diabetes mellitus and the colon. Curr Treat Options Gastroenterol 2017;15:460-474.

9. Yarandi SS, Srinivasan S. Diabetic gastrointestinal motility disorders and the role of enteric nervous system: current status and future directions. Neurogastroenterol Motil 2014;26:611-624.

10. Taylor C, Schubert ML. Decreased efficacy of polyethylene glycol lavage solution (Golytely) in the preparation of diabetic patients for outpatient colonoscopy: a prospective and blinded study. Am J Gastroenterol 2001;96:710-714.

11. Izzy M, Changela K, Alhankawi D, et al. Impact of glycemic control on colonoscopy outcomes: bowel preparation and polyp detection. Ann Gastroenterol 2016;29:332-335.

12. Kim YH, Seo EH, Lee JS, et al. Inadequate bowel cleansing efficacy of split-dose polyethylene glycol for colonoscopy in type 2 diabetic patients: a prospective and blinded study. J Clin Gastroenterol 2017;51:240-246.

13. Lankalapalli D, Kim M, Srivastava P, et al. Assessing the link between colonoscopy preparation quality and diabetes mellitus. Am J Gastroenterol 2017;5:22-25.

14. Ozturk NA, Gokturk HS, Demir M, Unler GK, Gur G, Yilmaz U. Efficacy and safety of sodium phosphate for colon cleansing in type 2 diabetes mellitus. South Med J 2010;103:1097-1102.

15. Sharma T, Das N, Ismail B, Castro-Pavia F, Cabral J, Villabona C. Evaluation of the effect of GLP-1 agonists on quality of bowel preparation for colonoscopy in patients with diabetes. Pract Diabetes 2017;34:167-168.

16. Alvarez-Gonzalez MA, Flores-Le Roux JA, Seoane A, et al. Efficacy of a multifactorial strategy for bowel preparation in diabetic patients undergoing colonoscopy: a randomized trial. Endoscopy 2016;48:1003-1009.

17. Grigg E, Schubert MC, Hall J, et al. Lubiprostone used with polyethylene glycol in diabetic patients enhances colonoscopy preparation quality. World J Gastrointest Endosc 2010;2:263-267.

18. Banerjee R, Chaudhari H, Shah N, Saravanan A, Tandan M, Reddy DN. Addition of lubiprostone to polyethylene glycol (PEG) enhances the quality \& efficacy of colonoscopy preparation: a randomized, double-blind, placebo controlled trial. BMC Gastroenterol 2016;16:133.

19. Stengel JZ, Jones DP. Single-dose lubiprostone along with split-dose PEG solution without dietary restrictions for bowel cleansing prior to colonoscopy: a randomized, double-blind, placebo-controlled trial. Am J Gastroenterol 2008;103:2224-2230.

20. Sofi AA, Nawras AT, Pai C, Samuels Q, Silverman AL. Lubiprostone plus PEG electrolytes versus placebo plus PEG electrolytes for outpatient colonoscopy preparation: a randomized, double-blind placebo-controlled trial. Am J Ther 2015;22:105-110.

21. Hayes A, Buffum M, Hughes J. Diabetic colon preparation comparison study. Gastroenterol Nurs 2011;34:377-382.

22. A-Rahim YI, Falchuk M. Bowel preparation before colonoscopy in adults. UpToDate 2021. Available from: https://www.uptodate. com/contents/bowel-preparation-before-colonoscopy-in-adults [Accessed 15 February 2021].

23. Manabe N, Cremonini F, Camilleri M, Sandborn WJ, Burton DD. Effects of bisacodyl on ascending colon emptying and overall colonic transit in healthy volunteers. Aliment Pharmacol Ther 2009;30:930-936.

24. Bharucha AE, Low P, Camilleri M, et al. A randomised controlled study of the effect of cholinesterase inhibition on colon function in patients with diabetes mellitus and constipation. Gut 2013;62:708-715.

25. Panarese A. Bowel preparation in diabetic patients undergoing colonoscopy. Endoscopy 2017;49:202.

26. Teng TY, Khor SN, Kailasam M, Cheah WK, Lau CC. Morning colonoscopies are associated with improved adenoma detection rates. Surg Endosc 2016;30:1796-1803.

27. Sanaka MR, Deepinder F, Thota PN, Lopez R, Burke CA. Adenomas are detected more often in morning than in afternoon colonoscopy. Am J Gastroenterol 2009;104:1659-1664.

28. Sanaka MR, Shah N, Mullen KD, Ferguson DR, Thomas C, McCullough AJ. Afternoon colonoscopies have higher failure rates than morning colonoscopies. Am J Gastroenterol 2006;101: 2726-2730.

29. Lee A, Iskander JM, Gupta N, et al. Queue position in the endoscopic schedule impacts effectiveness of colonoscopy. Am J Gastroenterol 2011;106:1457-1465.

30. Freedman JS, Harari DY, Bamji ND, et al. The detection of premalignant colon polyps during colonoscopy is stable throughout the workday. Gastrointest Endosc 2011;73:1197-1206.

31. Leffler DA, Kheraj R, Bhansali A, et al. Adenoma detection rates vary minimally with time of day and case rank: a prospective study of 2139 first screening colonoscopies. Gastrointest Endosc 2012;75:554-560.

32. Lurix E, Hernandez AV, Thoma M, Castro F. Adenoma detection rate is not influenced by full-day blocks, time, or modified queue position. Gastrointest Endosc 2012;75:827-834.

33. Diabetes and surgery. Clin Diabetes 2001;19:96-196.

34. Joshi GP, Chung F, Vann MA, et al; Society for Ambulatory Anesthesia. Society for Ambulatory Anesthesia consensus statement on perioperative blood glucose management in diabetic patients undergoing ambulatory surgery. Anesth Analg 2010;111:1378-1387.

35. Barker P, Creasey PE, Dhatariya K, et al; Membership of the Working Party. Peri-operative management of the surgical patient with diabetes 2015: Association of Anaesthetists of Great Britain and Ireland. Anaesthesia 2015;70:1427-1440.

36. Hochberg I, Segol O, Shental R, Shimoni P, Eldor R. Antihyperglycemic therapy during colonoscopy preparation: A review and suggestions for practical recommendations. United European Gastroenterol J 2019;7:735-740.

37. Practice guidelines for preoperative fasting and the use of pharmacologic agents to reduce the risk of pulmonary aspiration: application to healthy patients undergoing elective procedures: an updated report by the American Society of Anesthesiologists task force on preoperative fasting and the use of pharmacologic agents to reduce the risk of pulmonary aspiration. Anesthesiology 2017;126:376-393.

38. Hilsden RJ, Bridges R, Dube C, Heitman SJ, Rostom A. Scheduling rules for patients with diabetes mellitus that facilitate split-dosing improve the quality of bowel preparation for colonoscopy. PLoS One 2017;12:e0182225. 